\title{
Distribution of microplastics in mulched soil in Xinjiang, China
}

\author{
Can $\mathrm{Hu}^{1,2}$, Bing $\mathrm{Lu}^{2}$, Wensong Guo ${ }^{1}$, Xiuying Tang ${ }^{2}$, Xufeng Wang ${ }^{1 *}$, Yinghao Xue ${ }^{3 *}$, \\ Long Wang ${ }^{1,2}$, Xiaowei $\mathrm{He}^{1,2}$ \\ (1. College of Mechanical and Electrical Engineering, Tarim University, Alar 843300, Xinjiang, China; \\ 2. College of Engineering, China Agricultural University, Beijing 100083, China; \\ 3. Rural Energy and Environment Agency, Ministry of Agriculture and Rural Affairs, Beijing 100125, China)
}

\begin{abstract}
In order to study the distribution of soil microplastics in the plastic film mulched farmland, the fluidization-centrifugation secondary density flotation method was optimized and improved to obtain the samples. The main components of microplastics were analyzed by Fourier spectrometer, and the surface morphology, porosity, particle size and abundance distribution characteristics of microplastics were studied by electron microscopy. The results showed that plastic mulch is the main source of microplastics. Its morphology mainly consists of fragments, fibers and particles. The size of debris microplastics was larger, with an average of $1.6300 \mathrm{~mm}$, mainly distributed in the $0-300 \mathrm{~mm}$ cultivation layer while the size of particle micro plastic was smaller, with an average of $0.1400 \mathrm{~mm}$. The width of fiber microplastic was $5-20 \mu \mathrm{m}$, but the length could reach $0.2000-2.0000 \mathrm{~mm}$, with an average particle size of $0.9200 \mathrm{~mm}$. Fiber and granular microplastics could be seen in each soil layer. The surface layer of microplastics has a large number of pores characterized with a length of $50 \mu \mathrm{m}$ and a width of about 5-10 $\mu \mathrm{m}$, which might be an easy oxidation site for microplastics. This oxidation is continuous, making microplastics continuously decompose into smaller particles. The abundance of microplastics is negatively correlated with soil depth. The average abundance of microplastics is $161.50 \pm 5.20$ pieces/100 $\mathrm{g}$ in 0-300 $\mathrm{mm}$ soil layer, which is their main enrichment area. However, the average abundance of microplastics decreases to $11.20 \pm 1.10$ pieces/100 $\mathrm{g}$ in $400-800 \mathrm{~mm}$ soil depth. Moreover, the average particle size of microplastics is also linearly negatively correlated with soil depth. Microplastics with smaller particle size are easier to migrate as they pass through soil pores under the action of water and fertilizer. The research can provide s reference for understanding plastic mulch pollution.
\end{abstract}

Keywords: soil microplastics, plastic pollution, distribution, composition

DOI: $10.25165 /$ j.ijabe.20211402.6165

Citation: Hu C, Lu B, Guo W S, Tang X Y, Wang X F, Xue Y H, et al. Distribution of microplastics in mulched soil in Xinjiang, China. Int J Agric \& Biol Eng, 2021; 14(2): 196-204.

\section{Introduction}

Nowadays humans live in a world of plastics. All kinds of plastic wastes from industry and agriculture are decomposed and continuously refined into tiny plastic particles ${ }^{[1]}$, which has an adverse effect on environment. Internationally, plastic fragments with particle size less than $5 \mathrm{~mm}$ are usually defined as 'microplastics ${ }^{\text {,2] }}$. After the in-depth study of water microplastics, scholars are now paying more and more attention on the influence of residual microplastics in soil. The particle size of microplastics in soil is very small, mainly within micron or nano-scale range.

\section{Received date: 2020-09-17 Accepted date: 2021-01-20}

Biographies: Can Hu, PhD candidate, Associate Professor, research interests: prevention and control of residual film pollution, Email: hucanboy1@qq.com; Bing Lu, PhD candidate, Researcher, research interests: intelligent agricultural equipment, Email: 1170027843@qq.com; Wensong Guo, PhD candidate, Associate Professor, research interests: intelligent agricultural equipment, Email: 541577947@qq.com; Xiuying Tang, PhD, Professor, research interests: intelligent agricultural equipment, Email: txying@ cau.edu.cn; Long Wang, PhD candidate, Lecturer, research interests: soil machinery, Email: wangls11987@ qq.com; Xiaowei He, PhD candidate, Lecturer, research interests: soil machinery, Email:623685259@qq.com.

*Corresponding author: Xufeng Wang, PhD, Professor, research interests: agricultural equipment. College of Engineering, Tarim University, Alar 843300, Xinjiang, China. Tel: +86-13779803762, Email: wxfwyq@126.com; Yinghao Xue, Associate Researcher, research interests: agricultural ecological environment protection. Ministry of Agriculture and Rural Affairs, Beijing 100125, China. Email: xueyinghao@agri.gov.cn.
Microplastics in soil are likely to be swallowed by animals and indirectly enter human body, thus affecting the entire ecological chain $^{[3]}$. A large amount of microplastics residue can release harmful gases and affect the agricultural environment. Microplastics easily adhere to pollutants such as heavy metals and thus have an impact on the crop roots ${ }^{[4]}$. In addition, the surface of microplastics easily enriches microbial communities, thus affecting the soil ecosystem. Soil microplastics are mainly formed by agricultural mulching, organic fertilizers, pesticides and migration of various industrial microplastics. Among these, agricultural mulching films are one of the main sources of microplastics. China is the world's largest producer of plastic films. In 2019, China's plastic film output was 19.578 million t, from which the yield of agricultural mulching film was 2.4695 million $t$. The mulching area today exceeds 25 million $\mathrm{hm}^{2}$, accounting for $61 \%$ of the global agricultural mulch film coverage ${ }^{[5]}$. Plastic mulching in China is mainly practiced with cotton planting, in Northwest China. Xinjiang is the largest cotton planting base of Northwest China, where plastic mulch is most commonly used. In 2019, the total area of plastic mulch planting in the farmland of Xinjiang was about 3.8531 million $\mathrm{hm}^{2}$, with an average annual plastic film usage of about $235000 \mathrm{t}^{[6,7]}$. Plastic mulching increases temperature, preserves soil moisture and increases crop yield, and thus is an indispensable mean of agricultural production in arid regions of the world. However, it is difficult to recover the mulching film after weathering, which easily decomposes into plastic fragments and remain in the soil in form of flakes and strips 
with size ranging from $4-25 \mathrm{~cm}^{2}$. These are referred as plastic residual films ${ }^{[8]}$. Residual film is a kind of polyethylene polymer, which is difficult to recover. A large amount of plastic residual film remains in fields and is decomposed into secondary microplastics through the action of light, temperature, agricultural activities, soil microorganisms, etc. It is the main source of microplastics in soil ${ }^{[9]}$. The soil environment is complex and due to the lack of standardized microplastics separation and sampling methods, it is difficult to extract microplastics samples from the soil. This has resulted in a lack of research on the distribution form and abundance characteristics of microplastics in long-term mulched farmlands ${ }^{[10]}$. Studying the distribution characteristics of microplastics in such farmland soils can help to reveal the source of microplastics, changes that occur under mulching conditions, and better understand the ecological significance of mulching. This could provide an ecological assessment reference for global agricultural mulching areas.

The problem of soil microplastics has attracted increasing attention from international scholars. In 2012, Rillig ${ }^{[11]}$ raised the issue of microplastics in soil. Antunes et al. ${ }^{[12]}$ observed that the aged micro-plastic surface adsorbed more persistent organic pollutants (polychlorinated biphenyls and dichlorodiphenyltrichloroethane) in the soil. This might be related to the porous morphology of the micro-plastics that gives them an adhesive property after aging, attracting various substances from the soil. Eg et al. ${ }^{[13]}$ believed that the main source of microplastics was the micro or even nano-scale microplastics formed by continuous photolysis of agricultural mulching films and of greenhouse materials. Wartini et al. ${ }^{[14]}$ proposed a classification model for detecting the abundance of microplastics in soil by using near infrared spectroscopy, and established a regression model for predicting the abundance of microplastics. However, the recognition accuracy of such models at different soil conditions is quite different. By Fourier Transform Infrared Spectrometer (FTIR), 206 microplastics were found in a mulching free farmland in southeastern Germany by Piehl et al. ${ }^{[15]}$, indicating that microplastics in farmland can migrate and diffuse. Zhu et al. ${ }^{[16]}$ also believed that the aging of polymers such as, high density polyethylene, polyvinyl chloride and polystyrene, was the main reason for microplastic adsorption. Unlike larger plastic fragments visible to the naked eye, microplastics can continuously migrate and diffuse in the soil, releasing harmful gases ${ }^{[17]}$ and resulting in greater potential hazards ${ }^{[18]}$. Studying the basic distribution characteristics of microplastics can thus help with controlling plastic pollution by giving an insight about its main causes.

According to the existing literature ${ }^{[19]}$, the distribution characteristics and abundance data of microplastics in mulched farmlands are rarely reported, instead more scholars have paid attention to the problem of larger plastic residual film pollution in farmland soil ${ }^{[20-22]}$. Zhang et al. ${ }^{[23]}$ sampled and investigated the distribution characteristics of large plastic residual film pollution in cotton fields, and quantitatively studied the spatial distribution of residual film in soil. He et al. ${ }^{[2]}$ studied the changes in plastic residual film in a farmland in Xinjiang. They believed that the changes in the amount of residual film in cotton fields was due to the migration of smaller residual film fragments deeper into the soil layer. This downward migration of plastic residual film was due to the effect of water and the fertilizers by drip irrigation. It was inferred that residual film fragments were the main source of Xinjiang farmland microplastics ${ }^{[25]}$. All the above studies showed the possibility of microplastics in mulched farmlands in Xinjiang.
However, the current research is limited to the pollution by large visible plastics, while the distribution characteristics such as abundance and shape of microplastics in soil have not been studied. The representative plastic mulched crop in the current study is cotton.

In this research, the plastic mulched cotton field in Xinjiang was selected as the research object. The main contents of this study are as follows: (1) The micro plastic samples from different soil layers in cotton field were extracted to analyze the surface characteristics of soil microplastics, such as the overall morphology, pores, etc. (2) According to the statistics of the size and abundance of microplastics in different soil layers, the distribution law of different particle size and abundance was obtained. (3) Finally, the differences of particle size and abundance of microplastics in different soil layers were analyzed, and the main reasons for the formation of microplastics from plastic film fragments were revealed. This study determines the basic distribution characteristics and abundance migration changes of microplastics in mulched farmlands, evaluates the impact of plastic pollution with mulching, provides scientific reference for the ecological significance of mulching, and provides theoretical support for reducing the accumulation of microplastics and replacing mulching methods.

\section{Materials and methods}

\subsection{Experimental materials}

The soil samples used in the experiment were collected from Happy Farm in Alar City, Xinjiang $\left(40^{\circ} 33^{\prime} \mathrm{N}, 81^{\circ} 10^{\prime} \mathrm{E}\right.$, elevation, $1031 \mathrm{~m})$. The farmland crop was cotton, which had been continuously planted using mulching for more than 10 years. The soil was arid and according to USDA soil taxonomy, this kind of soil is most common in farmlands of Xinjiang. The soil condition was sandy loam, in which the clay content was $15 \%-20 \%$, and the sand content was $75 \%-80 \%$. The soil moisture content was between $20 \%-25 \%$. The polyethylene blow molding agricultural mulching film with thickness greater than $0.01 \mathrm{~mm}$ and a weathering period greater than $180 \mathrm{~d}$ was used in this farmland. The sample size of cotton fields selected were 20 with a maximum area of $30 \mathrm{hm}^{2}$ and minimum area of $4 \mathrm{hm}^{2}$. The sample data covered $800 \mathrm{hm}^{2}$. In each representative farmland, 5 plots were selected by 5 points method ( 5 plots were randomly selected according to plum blossom selection distribution method in each farmland). The size of each sample plot was $1 \mathrm{~m} \times 1 \mathrm{~m}$, and the depth of each sample plot was divided into 6 soil layers with a tillage depth of 0-300 $\mathrm{mm}$ (i.e., each soil layer is $50 \mathrm{~mm}$ deep, and 6 soil samples are taken from $0-300 \mathrm{~mm}$ soil layer). From each soil layer, $200 \mathrm{~g}$ of uniform soil samples were taken and the plastic fragments with particle size $>5 \mathrm{~mm}$ were separated. These fragments were packed in cotton cloth bags to prevent them mixing with the environment. In addition, in the five plots of farmland, each plot was divided into five soil layers with a depth of 300$800 \mathrm{~mm}$ (The depth of each soil layer was $100 \mathrm{~mm}$, and a total of 5 soil samples were taken from $300-800 \mathrm{~mm}$ soil layer). Again, $200 \mathrm{~g}$ of uniform soil samples from each soil layer were taken and the microplastics were separated and packed as described above. These were used to measure the infiltration and migration of microplastics. According to this calculation, from the sample depth of $0-800 \mathrm{~mm}$, a total of 11 samples were taken. Five samples were taken from each farmland and thus a total of 55 soil samples were collected. A total of 1100 soil samples were collected from 20 representative farmland cotton fields. 


\subsection{Experimental equipment}

Stereo microscope SteREO Discovery (V20, Carl Zeiss Company, Germany) which can read image size was used. Electronic balance $(0.0001 \mathrm{~g}$ precision, AR4201CN, Ohouse Company, USA). cold field emission scanning electron microscope (JSM-6700F, JEOL Company, Japan), gold spraying instrument (JFC-1600, JEOL Company, Japan), hot air drying oven (CJ, Shanghai Instrument, China), desktop intelligent high-speed freezing centrifuge (3H24RI, Hunan Hexi Instrument Equipment Co., Ltd, China), and ultrasonic cleaning machine (RY-9000-S, Shanghai Instrument, China) were used. The self-made fluidized-centrifugal secondary density flotation device, as shown in Figure 1, consisted of fluidized liquid forming device, fluidized flotation device, screening and filtering device, experimental bench and centrifugal treatment outlet. The sample was identified by Fourier transform infrared spectrometer (Thermo Fisher Nicoler iS10, Thermo Nicolet Company, USA).

\subsection{Test method}

\subsubsection{Microplastics sample separation device}

To separate microplastics, the fluidized density flotation method proposed by Nuelle et al. ${ }^{[26]}$ was improvised and a fluidized-centrifugal secondary density flotation device was designed (Figure 1). The basic method of this device was established by the optimization and improvement of density flotation and volumetric flask flotation method. The main source of microplastics in farmland soil is polyethylene material (PE) of agricultural mulch film which is decomposed into secondary microplastics after weathering. Its density is about 0.91$0.97 \mathrm{~g} / \mathrm{cm}^{3}$, which is relatively small. However, it is easy to obtain a higher recovery rate using density flotation. With fluidization-centrifugation secondary density flotation method, first of all, air induced overflow (AIO) was used to pre-extract the soil microplastics mixture on the basis of their fluidization in $\mathrm{NaCl}$ solution. Thereafter, the microplastic mixture was separated by centrifugation and the floating microplastic particles were retained by a filter screen. The specific operation method is as follows:

(1) Sample screening: The collected soil samples were air-dried in a drying box at $30^{\circ} \mathrm{C}$, and large particle soil blocks were filtered by a standard sieve of $5.0 \mathrm{~mm}$ (4 mesh). The weight of each sample after screening was $100 \mathrm{~g}$.

(2) Fluidization pre-selection: As shown in Figure 1, a certain volume of $\mathrm{NaCl}$ solution was taken in a bottle (bottle 1). The micro water pump 2 was turned on which allowed a part of $\mathrm{NaCl}$ solution to flow into the inner flotation bottle 5 at a certain flow rate. By the time, the micro water pump 2 was turned off, and the liquid was in a fluidized state. To this, $100 \mathrm{~g}$ of soil sample was quickly added to form a mixture. The $\mathrm{NaCl}$ solution generated an updraft, which caused lower density particles, such as microplastics, to float on the surface layer. The micro water pump 2 was turned on again which caused the $\mathrm{NaCl}$ solution to continuously flow into the outer bottle 4 and the floating particles formed the upper layer. This was the AIO method and results in fluidization preselection.

(3) Screening samples in the solution: A $10 \mu \mathrm{m}$ (1250 mesh) standard screen was used for filtration and the filtered samples were washed into an outer bottle with distilled water. These samples were dried at $50^{\circ} \mathrm{C}$ for $30 \mathrm{~min}$ and weighed to obtain sample quality.

(4) Centrifugal flotation of micro-plastic particles: The $3 / 4^{\text {th }}$ volume of the centrifuge tube, consistent with the quality of the sample, was filled with the sample and $\mathrm{NaCl}$ was used as the standard. After shaking, it was centrifuged in a high-speed freezing centrifuge at $2500 \mathrm{r} / \mathrm{min}$. After centrifugation for $5 \mathrm{~min}$, the floating material on the upper layer of the centrifuge tube was obtained again. After flushing the combined upper floats with distilled water, these were cleaned with an ultrasonic cleaning machine, and filtered with a mixed cellulose microporous filter membrane $(0.3 \mu \mathrm{m})$ to obtain the final micro-plastic sample. These were air dried in a petri dish and observed under a stereo microscope. The abundance, color and size of the particles were observed, excluding the non-plastic particles.

(5) Recovery rate of the device: The above steps were repeated until the microplastics from the soil were extracted and the soil was clean. This soil sample was dried at $80^{\circ} \mathrm{C}$. From the recovered microplastics, certain particles were selected for determining their weight and abundance statistics. These were used as recovery test samples. The weighed microplastic particles were added into clean soil, and then the fluidization-centrifugation secondary density flotation was carried out to recover these microplastics. After several steps of repeated recovery, the statistical recovery rate was $85.40 \%$. For the recovery test, 20 microplastic particles were added to $100 \mathrm{~g}$ clean soil, and the test was repeated. The micro plastic particles after recovery were calculated each time, and the average value after calculation was used to get the recovery rate of the test sample.

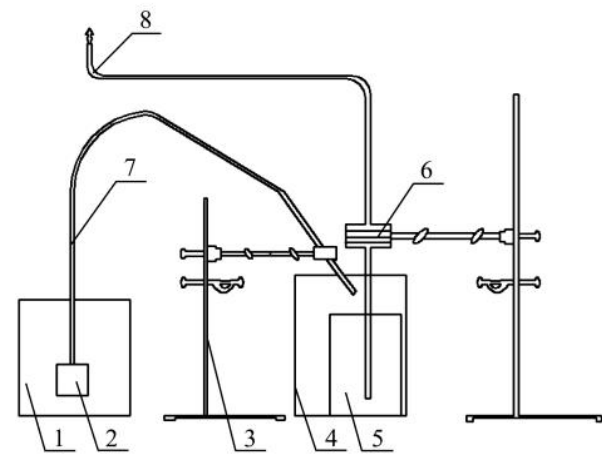

1. Sodium chloride solution bottle 2. Micro water pump 3. Test bench 4. Outer bottle (primary selection) 5 . Inner flotation bottle 6 . Screen inside the pipe 7. Quartz glass tube 8. Centrifugal treatment inlet

Figure 1 Schematic diagram of the centrifugal secondary density flotation device

\subsubsection{Abundance acquisition method}

The microplastic particles were identified and selected by stereo microscopy (visual inspection method). By adjusting the size of objective lens and eyepiece, the color, quantity, preliminary morphology and statistical abundance of the separated microplastics were identified in different soil layer samples. Abundance was represented as the content of microplastics in $100 \mathrm{~g}$ soil sample, expressed by the number of samples. It was defined as the number of microplastics per $100 \mathrm{~g}$ of soil, i.e., pieces/100 $\mathrm{g}^{[27,28]}$.

2.3.3 Statistical analysis of particle size

The approximate particle size was calculated through electron microscopy while the statistical analysis was done using stereo microscope. Previous literatures reported work on various forms of microplastics was referred to obtain a standard particle size reference ${ }^{[29]}$. The microplastics particle size from each soil layer was determined and the respective average value was obtained. This was used to develop a regression relationship between the change in particle size within different soil layers and within microplastics.

2.3.4 Electron microscopic analysis method

Scanning electron microscopy was used to determine the microstructural characteristics of microplastics. Since 
microplastics are dry and non-conductive, sample preparation was not required for electron microscopic scanning and only natural drying was sufficient. However, a prior cleaning of the sample by ultrasonic wave was required to remove impurities from the surface of microplastics. The naturally dried and cleaned sample was fixed on a conductive adhesive tape that was stuck on the SEM sample table. Gold spraying was done for $30 \mathrm{~s}$ using the gold spraying instrument set at a current of $20 \mathrm{~mA}$ and spraying time of $30 \mathrm{~s}$. After the sample table was placed in the sample chamber, the observation distance of the sample table was adjusted, and the microscopic characteristics of microplastics such as particle size, surface pores, surface cracking, profile and shape were observed.

\subsubsection{FTIR analysis}

FTIR was used to identify the components of the collected microplastics, so as to determine their source. Samples were randomly selected and processed according to a recent plastic identification protocol ${ }^{[30]}$. All spectral records were scanned for an average of 128 times with a resolution of $4 \mathrm{~cm}^{-1}$ and were compared with the commercial spectral libraries. The spectral data came from 'the combination technology of spectrum-chromatography-mass spectrometry and other spectral analysis of unknown substances' (BioRad KnowItAll Information System, Thermo Fisher Scientific Inc., USA).

\section{Results and analysis}

\subsection{Analysis of main components of microplastics}

The composition of microplastics from cotton field soil was analyzed by FTIR spectrum, and the results are shown in Figure 2. According to the contrast spectrum identification data, the main components of microplastics represent 6 characteristic peaks- the asymmetric stretching vibration peaks of $-\mathrm{CH}_{2}$ at $2918.36 \mathrm{~cm}^{-1}$, $2849.37 \mathrm{~cm}^{-1}, 1462.89 \mathrm{~cm}^{-1}, 1472.39 \mathrm{~cm}^{-1}$, and the in-plane rocking vibration peaks of $\left(\mathrm{CH}_{2}\right) n-(n \geq 4)$ at $719.46 \mathrm{~cm}^{-1}$ and $729.88 \mathrm{~cm}^{-1}$. By comparing FTIR spectral identification map data with the spectral data of polyethylene microplastics obtained by Zhang et al. and Corradini et al ${ }^{[31,32]}$, the results were found to be consistent. This revealed that the main component of agricultural mulching film from the cotton fields of Xinjiang might be polyethylene. Therefore, it can be inferred that a large amount of microplastics in cotton field soil come from the decomposition and fragmentation of agricultural mulching film residue.

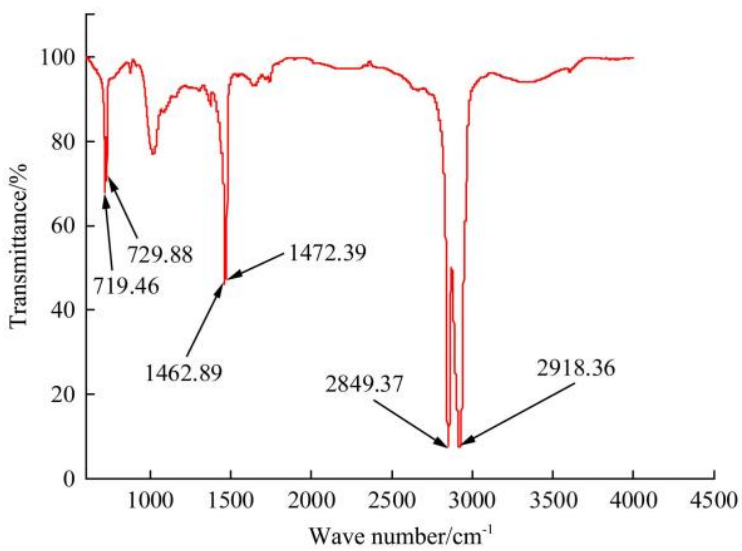

Note: The main components of microplastics showed six characteristic peaks, the asymmetric stretching vibration peaks of $-\mathrm{CH}_{2}$ - at $2918.36 \mathrm{~cm}^{-1}$, the asymmetric stretching vibration peaks of- $\mathrm{CH}_{2}$ - at $2849.37 \mathrm{~cm}^{-1},-\mathrm{CH}_{2-}$ at $1462.89 \mathrm{~cm}^{-1},-\mathrm{CH}_{2-}$ at $1472.39 \mathrm{~cm}^{-1}$, and the in-plane rocking vibration peaks with $\left(\mathrm{CH}_{2}\right) n-(n \geq 4)$ at 719.46 and $729.88 \mathrm{~cm}^{-1}$.

Figure 2 Fourier transform infrared characteristic spectra (FTIR spectra) of microplastics

\subsection{Surface morphological characteristics of microplastics}

3.2.1 Whole surface morphology characteristics of microplastics

Microplastics in cotton field soil have various forms and complex surface characteristics. Figure 3 shows micro-plastic particles separated from cotton field soil, of which larger particles are visible to the naked eye and can be directly identified as microplastics generated by fragmentation and decomposition of agricultural mulching film. Smaller particle of microplastics could not be recognized by naked eyes. Also, when observed through microscopic examination, there were a large number of microplastics with size $<5 \mathrm{~mm}$ in the soil. Among these, a fragment was defined as the microplastic with particle size $>0.05$ $\mathrm{mm}$, flaky length and width, the ratio of length to width of no more than $20 \%$, and the overall fragmentary shape. A particle was referred for those with size $<0.05 \mathrm{~mm}$. A fiber was referred to microplastics with long strip shape; length larger than the width with the ratio of width to length less than $10 \%$. Microscopic observation revealed that the surface of the microplastics was porous and ashing. Visual inspection revealed that the shapes of the microplastics were mainly fragments, particles and fibers. Fragments of microplastics were mainly distributed in $0-300 \mathrm{~mm}$ soil layer, while particles and fibers were observed in all soil layers.

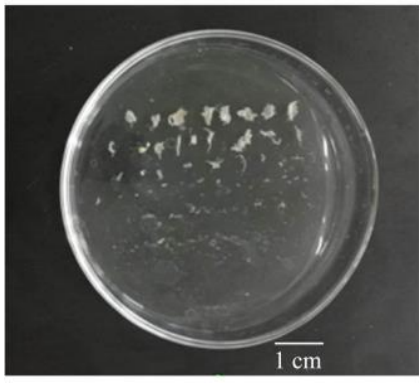

a. Different types of microplastics after separation

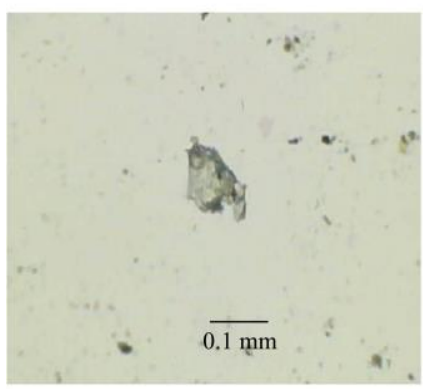

c. Particle microplastics

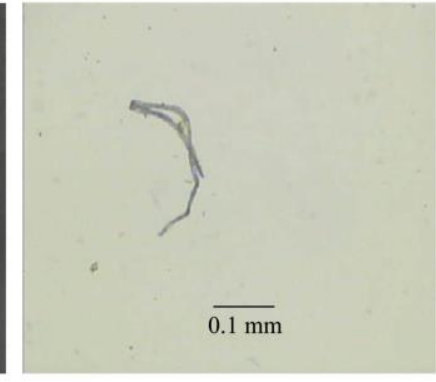

b. Fiber microplastics

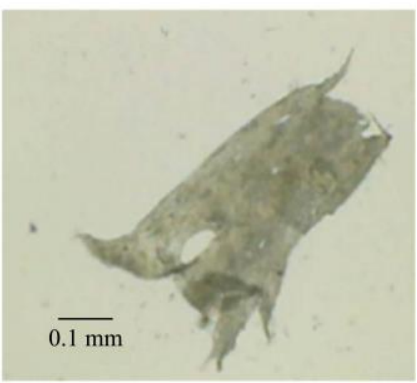

d. Fragmentary microplastics
Figure 3 Collected microplastic samples

Figure 4 shows 6 representative forms of samples observed under electron microscope. These were divided into fragment, particle and fiber microplastics. The edge of fragment microplastics was irregular in shape, which might be a result of weathering and decomposition of mulching film. Particle microplastics were rectangular with brittle pulverized edges. These edges were damaged and the surface was rough causing these microplastics to easily mix with other substances in the soil. This made it difficult to determine the source of these plastics. Fibrous microplastics were uneven and had no finished shape. The edges formed the shape of silk flocs. The overall surface morphology characteristics of the three types of microplastics were similar to the fiber morphology of thin film microplastics reported in a previous research ${ }^{[33]}$. 


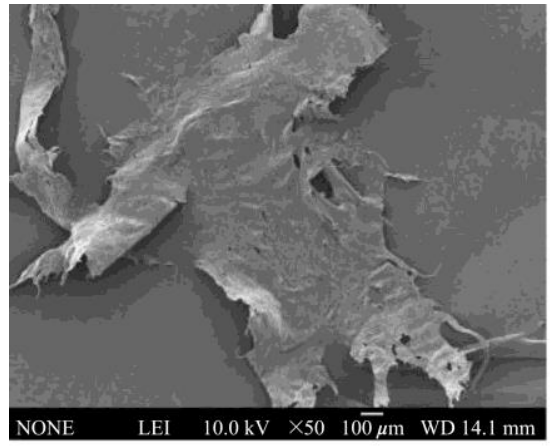

a. Fragmentary microplastics

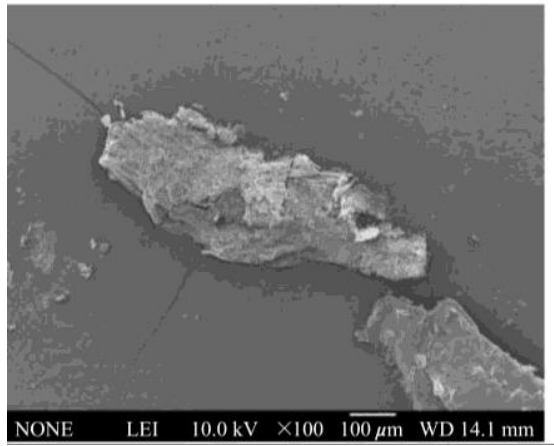

d. Granular microplastics from deeper soil layers

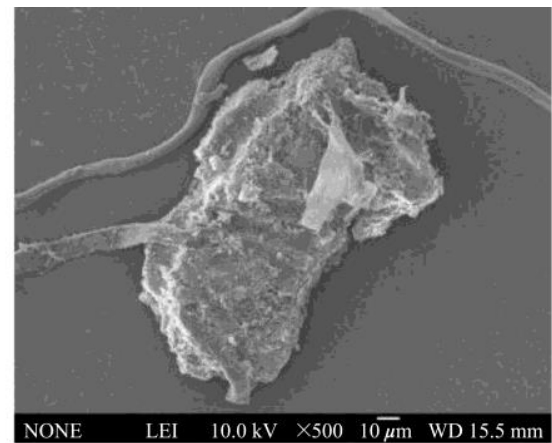

b. Particle microplastics

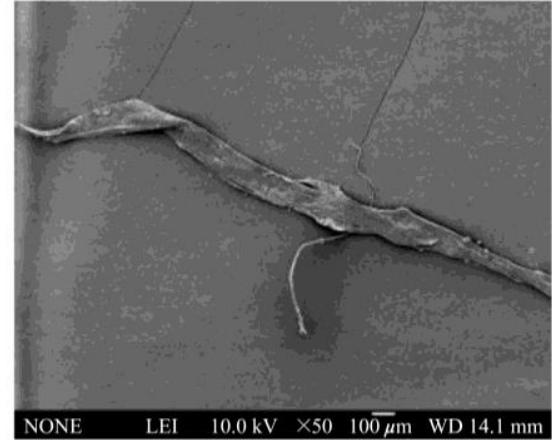

e. Fiber microplastics

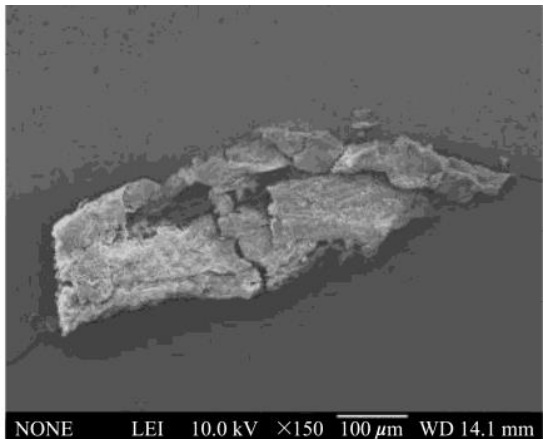

c. Granular microplastics from deeper soil layers

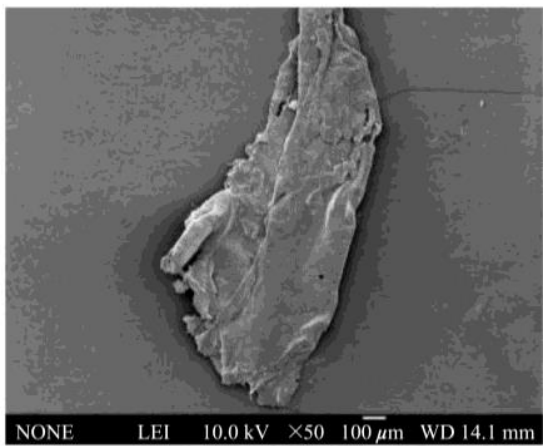

f. Overlapping fiber microplastics

Figure 4 Scanning electron micrograph of microplastics with different characteristics in soil

\subsubsection{Pore characteristics of microplastics surface}

Porosity is an important and apparent feature of microplastics, which affects their surface properties. The electron microscopic observations of the collected samples are shown in Figure 5. There are a number of pores on the surface of fragmented microplastics (Figure 5a), which is closely related to the weathering characteristics of the plastic film. The pores are probably formed due to the decomposition of the weathered plastic film. The surface layer was observed to have a number of fine particles adhered to it, which was not a smooth body characteristic, indicating that the fragmented microplastics have a certain adhesion. The edge of fragmented microplastics was like an irregular silk flocculent, which was like a shape caused by tearing due to an external mechanical force. Luo et al. ${ }^{[34]}$ also observed similar fragments formed due to tearing from plastic films subjected to a mechanical force. Therefore, the flocculent appearance might be because of the crushing and tearing during agricultural machinery operation and due to further weathering.

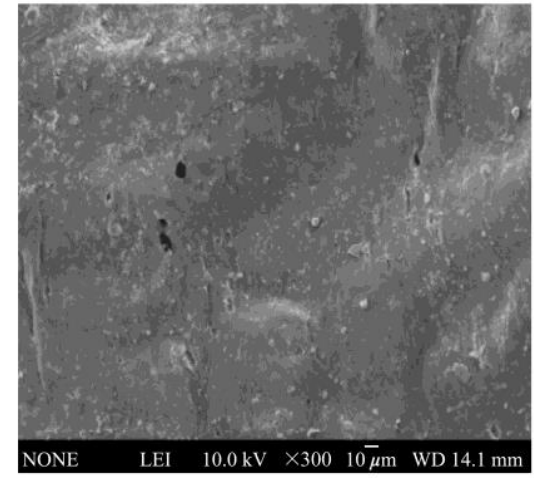

a. Surface pore characteristics of the fragmentary microplastics

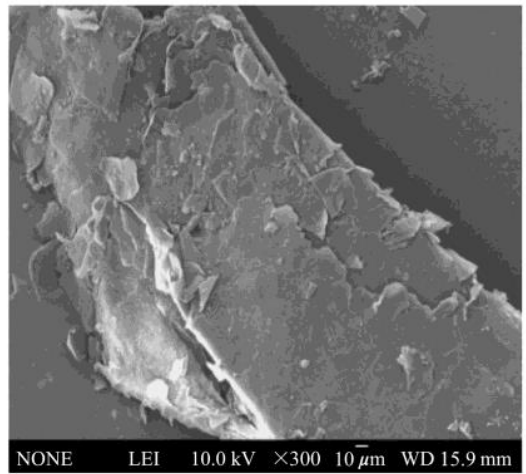

d. Surface fragmentation characteristics of particle microplastics

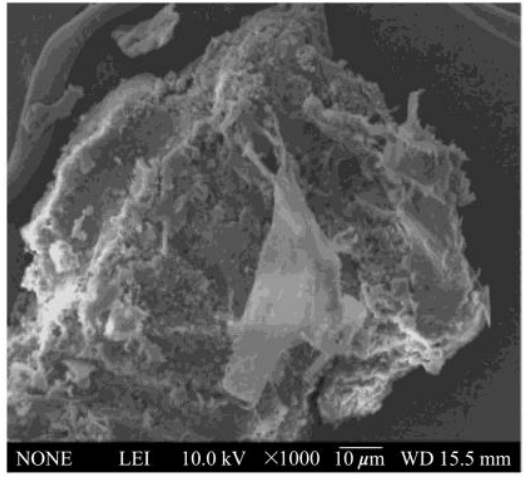

b. Local characteristics of particle microplastics

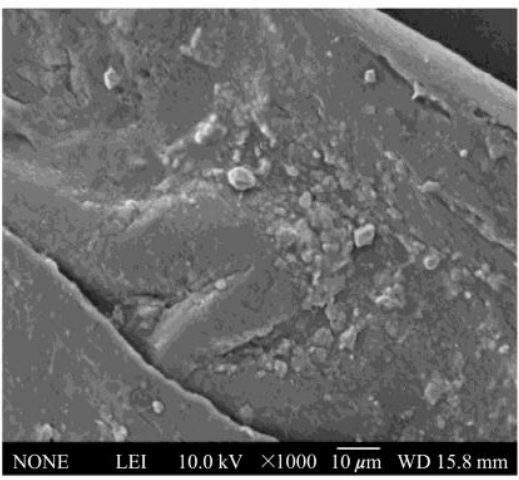

e. Uneven surface of fiber microplastics

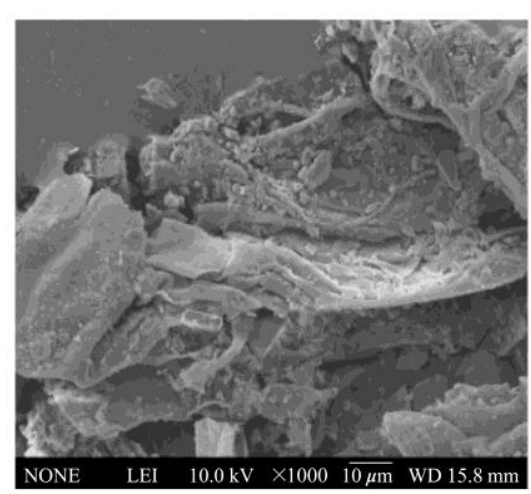

c. Local characteristics of particle microplastics

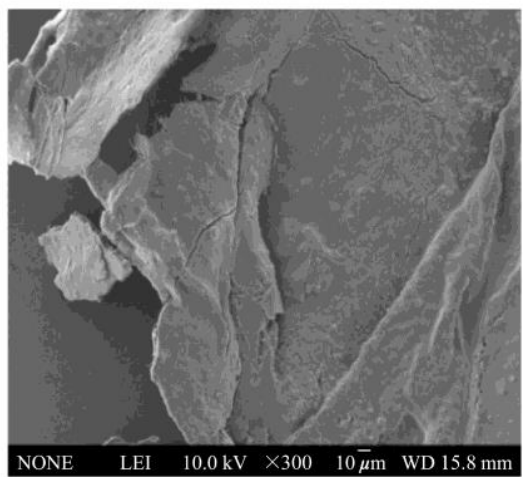

f. Local tear and other changes of fiber microplastics

Figure 5 SEM image of the local surface of microplastics with different characteristics in soil 
The granular microplastics were characterized by a large number of fine fragments and pulverized surface (Figures $5 \mathrm{~b}$ and $5 \mathrm{c})$, which indicates that the microplastics were fragmented under the influence of weathering. These characteristics were more prominent on some granular microplastics, almost covering the entire surface (Figure 5d), which may be due to the difference in weathering time. Most microplastics showed a large number of pores on their surface, including fine longitudinal pore cracks with a pore length of $>50 \mu \mathrm{m}$ and a width of about 5-10 $\mu \mathrm{m}$. The pore edges were irregular in distribution and displayed a complex structure. These pore characteristics might be caused by irregular tearing or weathering of microplastics. Considering the weathering characteristics of a plastic film, these are speculated to be the result of the continuous oxidation of microplastics. The pores on the surface layer of microplastics might be an easily oxidized zone, with continuous oxidation causing the microplastics to decompose into smaller particles. In addition, the porous characteristic allows an easy adherence to various other substances in the soil. Corcoran et al. ${ }^{[35]}$ also believed that the porous characteristics of microplastics surface was conducive to easy oxidation with pore edges being preferred oxidation sites. These can thus be used to identify the oxidation characteristics of microplastics.

Therefore, the change from plastic film to microplastics might involve the following process: After the plastic film is broken down by weathering or external mechanical force, it forms irregular edges, starts to oxidize and forms more pores. Soil substances adhere to the pores and enrich microbial communities on the surface. Continuous oxidation causes the pores expansion and thus decomposition into smaller and smaller microplastics.

The surface of fibrous microplastics was observed to be linear (Figures 5e and 5f). The characteristics similar to fragmented microplastics and more pore cracks were observed. The fibrous microplastics might be formed due to further breaking of the plastic film into silk flocculent after the action of agricultural machinery. Microplastics in soil have the characteristics of rough surface, porosity, pulverization and fission due to weathering. This kind of porous and rough surface increases the surface area of microplastics and increases the adsorption capacity of other pollutants in the soil.

\subsection{Particle size distribution characteristics of microplastics} in different soil layers

\subsubsection{Particle size range}

The particle size distribution of microplastics after statistical analysis is shown in Table 1. The particle size of microplastics in 0-300 $\mathrm{mm}$ soil layer was between $0.0310 \mathrm{~mm}$ and $4.9000 \mathrm{~mm}$, and the large fragments approached a maximum particle size of $5 \mathrm{~mm}$. Among these, the plastic fragments larger than $5 \mathrm{~mm}$ were removed and were not considered as microplastic particles. These could be seen with naked eyes. The average particle size of microplastics in 400-800 mm soil layer ranged from $0.0011 \mathrm{~mm}$ to $0.4080 \mathrm{~mm}$, which was clearly smaller. It was more difficult to obtain and observe these samples that could not be identified by naked eyes. There were small particles of microplastics in each layer of the soil. The minimum particle size in the collected samples was $0.0011 \mathrm{~mm}$, which was observed to be of particle type microplastic under the microscope. Limited by more sophisticated instruments, there might be nano-scale microplastics that could not be accurately detected.
Table 1 Size range of microplastics in different soil layers of a cotton field in Xinjiang, China

\begin{tabular}{cccc}
\hline $\begin{array}{c}\text { Serial } \\
\text { number }\end{array}$ & $\begin{array}{c}\text { Soil depth } \\
/ \mathrm{mm}\end{array}$ & $\begin{array}{c}\text { Particle size range } \\
/ \mathrm{mm}\end{array}$ & Shape category \\
\hline 1 & $0-50$ & $0.0420-4.6000$ & Fragments, fibers, particles \\
2 & $50-100$ & $0.0800-4.9000$ & Fragments, fibers, particles \\
3 & $100-150$ & $0.0310-4.6000$ & Fragments, fibers, particles \\
4 & $150-200$ & $0.0385-4.4000$ & Fragments, fibers, particles \\
5 & $200-250$ & $0.0470-4.0000$ & Fragments, fibers, particles \\
6 & $250-300$ & $0.0385-3.8000$ & Fragments, fibers, particles \\
7 & $300-400$ & $0.0150-1.8800$ & Fragments, fibers, particles \\
8 & $400-500$ & $0.0040-0.4080$ & Fibers, particles \\
9 & $500-600$ & $0.0026-0.2836$ & Fibers, particles \\
10 & $600-700$ & $0.0019-0.2801$ & Fibers, particles \\
11 & $700-800$ & $0.0011-0.2695$ & Fibers, particles \\
\hline
\end{tabular}

3.3.2 Particle size distribution of microplastics

The particle size distribution of microplastics is shown in Figure 6a. The particle size of debris microplastics was relatively larger, with minimum particle size of about $0.1400 \mathrm{~mm}$ in the collected samples and the maximum particle size close to $5.0000 \mathrm{~mm}$. The average particle size was $1.6300 \mathrm{~mm}$. The particle size of granular microplastics was small with a minimum size of $0.0011 \mathrm{~mm}$, and the average particle size of $0.1400 \mathrm{~mm}$. The fibers had a small width of 5-20 $\mu \mathrm{m}$, but their length could reach $0.2000-2.0000 \mathrm{~mm}$. Their average particle size was $0.9200 \mathrm{~mm}$.

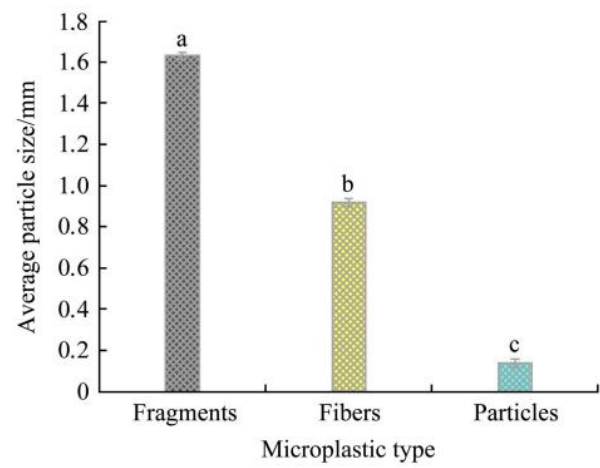

a. The average particle size distribution of the three types of microplastics

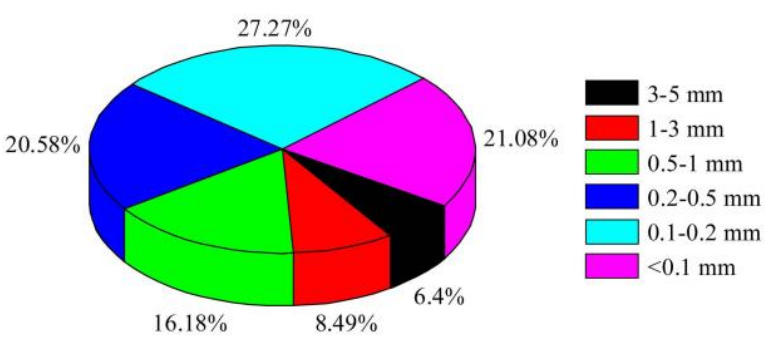

b. Proportion of microplastics with different particle sizes

Note: The average particle size of the fragment microplastics is significantly larger than that of the other two types.

Figure 6 Average particle size distribution of different microplastic types

The proportion of microplastics with different particle sizes is shown in Figure 6b. Within the samples collected from 0 to $800 \mathrm{~mm}$ soil layer, the proportion of $3-5 \mathrm{~mm}$ size particles was $6.39 \%$. The proportion of microplastics with $<0.5 \mathrm{~mm}$ particle size was the largest, accounting for $68.93 \%$. $0.2-0.5 \mathrm{~mm}$ size particles accounted for $20.58 \%, 0.1-0.2 \mathrm{~mm}$ size particles were $27.27 \%$, and the proportion of $<0.1 \mathrm{~mm}$ size particles was $21.08 \%$. As also observed from the particle size range distribution in Table 
1, the proportion of particles with smaller size is largest in deep soil. The average particle size of microplastics in each layer of soil was counted, and regression analysis was carried out according to different soil layers, as shown in Figure 7a. The results show that with the increase of soil depth, the average particle size of microplastics becomes smaller, and there is a linear negative correlation between them $\left(R^{2}=0.9457, p<0.1\right)$. This shows that microplastics with smaller particle sizes easily migrate to deeper soil, while microplastics with larger particle size do not migrate easily through soil voids. After further decomposition under natural conditions, the particle size becomes smaller and infiltration migration might occur.

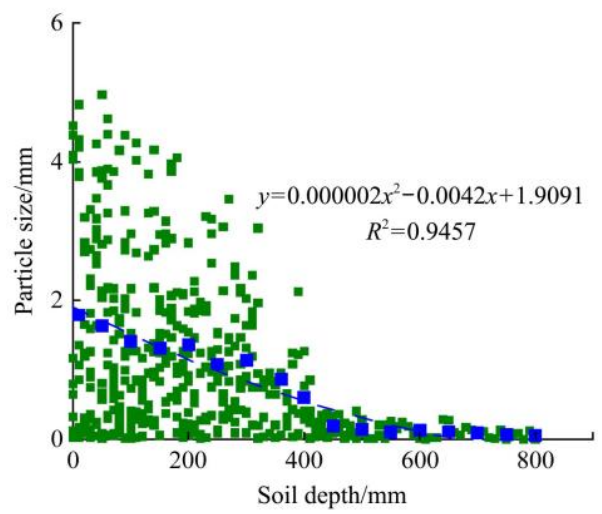

Note: each data point in the figure represented a microplastic in the soil layer measured.

a. Relationship between average particle size and soil depth.

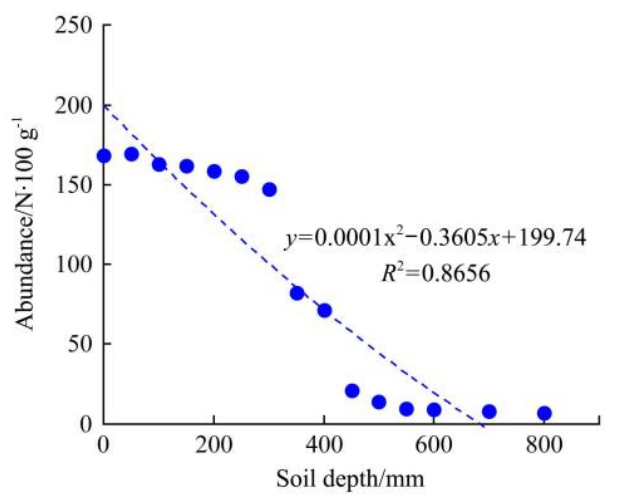

b. Relationship between microplastic abundance and soil depth

Figure 7 Relationship between microplastic and soil depth

\subsection{Abundance distribution characteristics of microplastics in different soil layers}

The abundance distribution characteristics of microplastics in different soil layers are shown in Figure 8. It can be concluded that the total amount of microplastics from long-term mulched cotton fields is mainly concentrated in $0-300 \mathrm{~mm}$ soil layer, with an average abundance of $161.50 \pm 5.20$ pieces $/ 100 \mathrm{~g}$. When the soil depth was less than $50 \mathrm{~mm}$, the average abundance was $172.40 \pm$ 6.83 pieces $/ 100 \mathrm{~g}$ with a particle size range of $0.0420-4.6000 \mathrm{~mm}$. When the depth of sampling soil was $600 \mathrm{~mm}$, the average abundance was $7.00 \pm 0.86$ pieces $/ 100 \mathrm{~g}$ with a particle size range of $0.0011-0.2695 \mathrm{~mm}$. In the deep layer of $400-800 \mathrm{~mm}$, the average abundance value decreased to $11.20 \pm 1.10$ pieces/100 g. The soil layer at $0-300 \mathrm{~mm}$ is the tillage layer of Xinjiang cotton fields. Affected by long-term tillage, plastic film fragments formed by mechanical operation, temperature, light and other factors are mainly concentrated in this tillage layer. Thus, the morphology, particle size and abundance of microplastics in the $0-300 \mathrm{~mm}$ soil layer are basically similar, which is also the main reason this layer is the main enrichment layer of microplastics. The abundance distribution relationship between different soil depth layers and microplastics is shown in Figure $7 \mathrm{~b}$. The abundance of microplastics in tillage layer $(0-300 \mathrm{~mm})$ was significantly higher than that in non-tillage layer $(300-800 \mathrm{~mm})$, and the abundance decreased linearly with soil depth $\left(R^{2}=0.8656, p<0.1\right)$. In order to confirm this result, repeated sampling from various spots of cotton fields was done. With the increase in soil depth, the abundance of microplastics decreased, the shape was mainly particles and fibers, and the particle size range was smaller.

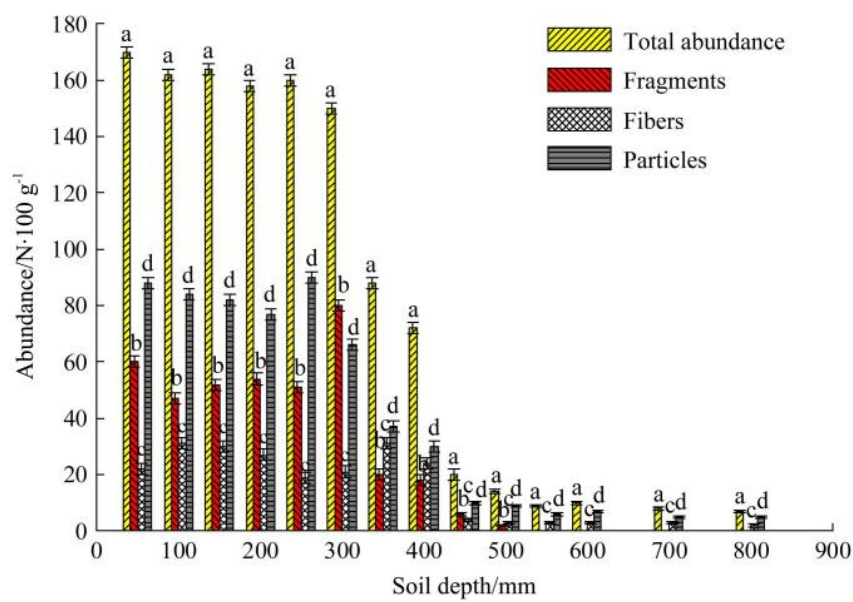

Figure 8 Abundance distributions of various microplastics in different soil layers

According to Xinjiang's farmland tillage characteristics ${ }^{[36]}$, $0-300 \mathrm{~mm}$ is the main tillage layer in cotton fields. The working depth of agricultural machinery and field agriculture is in this soil layer, which is also the main soil layer for microplastics after mulching. A certain amount of microplastics were also found in the $300-800 \mathrm{~mm}$ non-tillage layer which suggested that the upper microplastics migrated to the non-tillage layer.

According to the analysis of microplastics particle size distribution characteristics, it was found that $0-300 \mathrm{~mm}$ tillage layer is the main distribution area of visible microplastics debris. In 400-800 mm deep soil layer, debris microplastics with larger particle size gradually decreased. Thus, the more the soil depth was, the smaller the particle size of microplastics was. Microplastics can migrate and infiltrate into the deep soil along with the utilization of water and fertilizer. In this study, fiber and granular microplastics with a smaller particle size were mainly observed in non-tillage layer $(300-800 \mathrm{~mm})$ which indicated that these kinds of microplastics with smaller particle size were easier to penetrate into deep soil. Under the action of water and fertilizer irrigation in the soil, smaller microplastics permeate and migrate to deep soil through soil pores, while the debris microplastics having larger particle sizes and less probability of infiltration and migration remain concentrated in the upper layer. Therefore, microplastics in deep soil are mainly produced by infiltration and migration of fiber and particle microplastics. This kind of infiltration migration might be continuous, and there might also be infiltration migration of microplastics to deeper soil layers or even to groundwater layers, which requires more in-depth research.

\section{Conclusions}

The investigation of cotton fields in Xinjiang shows that long-term mulching in cotton fields causes the enrichment of soil microplastics, and these microplastics are mainly composed of PE. 
Under no other pollution sources, agricultural mulching is the main source of microplastics. Soil microplastics have complex morphological characteristics and can be divided into three types: fragments, particles and fibers. Among them, fragments have larger particle sizes and are mainly distributed in $0-300 \mathrm{~mm}$ soil tillage layer, while particles and fibers have smaller particle sizes and are distributed in all layers. Under the influence of tillage, such as water and fertilizer, these microplastics infiltrate and migrate to non-tillage layer $(300-800 \mathrm{~mm})$. There are a number of pores with length $>50 \mu \mathrm{m}$ and width about $5-10 \mu \mathrm{m}$ on the surface layer of microplastics, which are their oxidation sites causing the microplastics to decompose into smaller and smaller fragments. The abundance of microplastics in mulched cotton fields was negatively correlated with soil depth. The average abundance of microplastics in 0-300 mm soil layer was $161.50 \pm 5.20$ pieces $/ 100 \mathrm{~g}$, while in $400-800 \mathrm{~mm}$ soil layer, the average abundance decreased to $11.20 \pm 1.10$ pieces $/ 100 \mathrm{~g}$. Moreover, the average particle size of microplastics was also linearly negatively correlated with soil depth, with deeper soils mainly containing particles and fibers. This indicated that microplastics with smaller particle size might continuously migrate to the deeper soil and infiltrate in each multi-dimensional direction of soil depth, and there might also be migration and diffusion in deeper soils. The direction, rate and depth of migration need more in-depth research. In this study, cotton fields in Xinjiang Region of China were taken as the research object, and the causes and basic distribution patterns of microplastics in plastic mulched farmland were determined, which added a new understanding of the impact of plastic pollution caused by plastic mulching in global farmlands and provided a reference for the ecological significance of plastic mulching.

\section{Acknowledgments}

This work was supported by National Natural Science Foundation of China (Grant No. 32060288) and the Key R \& D Projects of Xinjiang Production and Construction Corps (Grant No. 2019AB007) and the Special Project for Agricultural Ecological Environment Protection of Ministry of Agriculture and Rural Areas of China (2110402).

\section{[References]}

[1] Geyer R, Jambeck J R, Law K L. Production, use, and fate of all plastics ever made. Advanced Science, 2017; 3(7): e1700782.

[2] Liu M T, Lu S B, Song Y, Lei L, Hu J, Lv W W, et al. Microplastic and mesoplastic pollution in farmland soils in suburbs of Shanghai, China. Environmental Pollution, 2018; 242: 855-862.

[3] Zhang G S, Liu Y F. The distribution of microplastics in soil aggregate fractions in southwestern China. Science of the Total Environment, 2018; 642: 12-20.

[4] Rillig M C, Bonkowski M. Microplastic and soil protists: A call for research. Environmental Pollution, 2018; 241: 1128-1131.

[5] Hu C, Wang X F, Wang S, Lu B, Guo W, Liu C, et al. Impact of agricultural residual plastic film on growth and yield of drip-irrigated cotton in arid region of Xinjiang, China. International Journal of Agricultural and Biological Engineering, 2020; 13(1): 160-169.

[6] Hu C, Wang X, Chen X G, Tang X, Zhao Y, Yan C R. Study on the status quo of farmland residual film pollution in Xinjiang and its prevention and control strategy. Transactions of the CSAE, 2019; 35(24): 223-234. (in Chinese)

[7] Huang Y, Liu Q, Jia W Q, Yan C R, Wang J. Agricultural plastic mulching as a source of microplastics in the terrestrial environment. Environmental Pollution, 2020; 260: 114096.

[8] Yan C R, Mei X, He W Q, Zheng S. Present situation of residue pollution of mulching plastic film and controlling measures. Transactions of the CSAE, 2006; 22(11): 269-272. (in Chinese)
[9] Kim D, Chae Y, An Y J. Mixture toxicity of nickel and microplastics with different functional groups on daphnia magna. Environmental Science \& Technology, 2017; 51(21): 12852-12858.

[10] Ren X W, Tang J C, Yu C, He J. Advances in research on the ecological effects of microplastic pollution on soil ecosystems. Journal of Agro-Environment Science, 2018; 37(6): 1045-1058. (in Chinese)

[11] Rillig M C. Microplastic in terrestrial ecosystems and the soil Environmental Science \& Technology, 2012; 46: 6453-6454.

[12] Antunes J C, Frias J G L, Micaelo A C, Sobral P. Resin pellets from beaches of the portuguese coast and adsorbed persistent organic pollutants. Esuarine Coastal and Shelf Science, 2013; 130: 62-69.

[13] Ng E-L, Lwanga E H, Eldridge S M, Johnston P, Hu H-W, Geissen V, et al An overview of microplastic and nanoplastic pollution in agroecosystems. Science of the Total Environment, 2018; 627: 1377-1388.

[14] Wartini N, Budiman M, McBratney A. Convolutional neural network for soil microplastic contamination screening using infrared spectroscopy. Science of the Total Environment, 2020; 702: 1-9.

[15] Piehl S, Leibner A, Löder M G J, Dris R, Bogner C, Laforsch C Identification and quantification of macro- and microplastics on an agricultural farmland. Sci Rep-UK, 2018; 8(1): 17950.

[16] Zhu Y G, Zhu D, Xu T, Ma J. Impacts of (micro) plastics on soil ecosystem: Progress and perspective. Journal of Agro-Environment Science, 2019; 38(1): 1-6. (in Chinese)

[17] Nicole B, Verena W, Volke W. Contaminant release from aged microplastic. Environmental Chemistry, 2017; 14(6): 394-405.

[18] Nizzetto L, Langaas S, Futter M. Pollution: Do microplastics spill on to farm soils? Nature, 2016; 537: 488.

[19] Chae Y, An Y J. Current research trends on plastic pollution and ecological impacts on the soil ecosystem: A review. Environmental Pollution, 2018; 240: 387-395.

[20] Yan C R, Wang X, He W Q, Ma H, Cao S, Zhu G F. Study on the residue of plastic film in cotton field in Shihezi, Xinjiang. Acta Ecologica Sinica, 2008; 28(7): 3470-3474. (in Chinese)

[21] He W Q, Yan C R, Liu S, Chang R, Wang X, Cao S, et al. The use of plastic mulch film in typical cotton planting regions and the associated environmental pollution. Journal of Agro-Environment Science, 2009; 28(8): 1618-1622.

[22] Yan C R, He W Q, Turner N C. Plastic-film mulch in Chinese agriculture: Importance and problems. World Agriculture, 2014; 4(2): 32-36.

[23] Zhang D, Liu H B, Hu W L, Qin X H, Ma X W, Yan C R, et al. The status and distribution characteristics of residual mulching film in Xinjiang, China. Journal of Integrative Agriculture, 2016; 15: 2639-2646.

[24] He H J, Wang Z H, Li G, Zheng X, Zhang J Z, Li W, et al. Distribution characteristics of residual film over a cotton field under long-term film mulching and drip irrigation in an oasis agroecosystem. Soil \& Tillage Research, 2018; 180(1): 194-203.

[25] Ruimin Q, Davey L J, Zhen L, Qin L, Changrong Y. Behavior of microplastics and plastic film residues in the soil environment: A critical review. Science of the Total Environment, 2020; 703: 1-13.

[26] Nuelle M T, Dekiff J H, Remy D, Fries E. A new analytical approach for monitoring microplastics in marine sediments. Environmental Pollution, 2014; 184: 161-169.

[27] Dris R, Gasperi J, Rocher V, Tassin B. Synthetic and non-synthetic anthropogenic fibers in a river under the impact of Paris Megacity: Sampling methodological aspects and flux estimations. Science of the Total Environment, 2018; 618: 157-164.

[28] Luo W, Su L, Craig N J, Du F, Wu C, Shi H. Comparison of microplastic pollution in different water bodies from urban creeks to coastal waters. Environmental Pollution, 2019; 246: 174-182.

[29] O'connor D, Pan S, Shen Z, Song Y, Jin Y, Wu W M, et al. Microplastics undergo accelerated vertical migration in sand soil due to small size and wet-dry cycles. Environmental Pollution, 2019; 249: 527-534.

[30] Li J, Qu X, Su L, Zhang W, Yang D, Kolandhasamy P, et al Microplastics in mussels along the coastal waters of China. Environmental Pollution, 2016; 214: 177-184.

[31] Zhang M J, Zhao Y, Qin X, Jia W Q, Chai L W, Muke H, et al. Microplastics from mulching film is a distinct habitat for bacteria in farmland soil. Science of the Total Environment, 2019; 688: 470-478.

[32] Corradini F, Bartholomeus H, Huerta L E, Gertsen H, Geissen V. Predicting soil microplastic concentration using vis-NIR spectroscopy. Science of the Total Environment, 2019; 650: 922-932.

[33] Zhou Q, Zhang H B, Zhou Y, Li Y, Xue Y, Fu C C, et al. Separation of microplastics from a coastal soil and their surface microscopic features. 
Chinese Science Bulletin, 2016; 61: 1604-1611. (in Chinese)

[34] Luo K, Yuan P, Jin W, Yan J S, Bai S, Zhang C, et al. Design of chain-sieve type residual film recovery machine in plough layer and optimization of its working parameters. Transactions of the CSAE, 2018; 34(19): 19-27. (in Chinese)
[35] Corcoran P L, Biesinger M C, Grifi M. Plastics and beaches: A degrading relationship. Marine Pollution Bulletin, 2009; 58: 80-84.

[36] Zhao Y, Chen X G, Wen H J, Zhen X, Niu Q, Kang J. Research status and prospect of control technology for residual plastic film pollution in farmland. Transactions of the CSAM, 2017; 48(6): 1-12. (in Chinese) 